\title{
Unique Key Using Encryption and Decryption of Image
}

\author{
Kaladharan N \\ Assistant Professor, Department of Electrical and Electronics Engineering, Annamalai University, India
}

\begin{abstract}
Image data security is the essential portion in communication and multi media world. During storing and sharing, avoid third party access of data is the challenge one. Providing security of data is the clever work and art also. Many protection algorithms are used in recent years. Protection may be given of a data is converting the original in to some unknown form, signals, sketch etc., which is not understand by any one. Cryptography is the best technique of image data security. In Greek, crypto refers 'hidden' and graphy refers 'script'. Cryptography has two processes namely encryption and decryption. Encryption achieves the conversion by possessing a key of original data into unreadable form called encoding. Restoring of encrypted data in to original is decoding or decryption. Key, code or password is the vital role in cryptography. This paper presents the performance of encryption and decryption of an image using a single key algorithm and tested on some images and shows fine results.
\end{abstract}

General terms: Cryptography, Security, Encoding, Decoding

Keywords: Image processing, Encryption, decryption, key, password

\section{INTRODUCTION}

Image data are frequently shared and stored in worldwide at various end to end. Images may contain highly confidential and responsive informations [1]. Image type of data are particularly used in the area of military wing, forensic department, intelligent agencies, medicine researches, government sectors, multimedia, film division etc.,[4][6].During their transmission, the data can be accessed illegally and misuse by the hackers and unauthorized users[7]. These troubles are usually happened in the internet communication [12]. Hence data needs high protection on consistently. Ensure high protection of data, cryptography is the suitable technique keeps the data as safe as in transfer [11]. Main reason behind using cryptography is authentication, secrecy, non disclaimer, consistency and honesty at any instant of data transfers. Cryptography can be describe as the skill of protection file and it makes sure that only the related people to access the content [10]. This paper presents cryptography method using encryption and decryption of a single key algorithm with some image data.

This paper is organized as follows, Section II describes cryptography method, Section III represent proposed scheme, section IV express implementation algorithm Section V shows the experimental results and Section VI deals the conclusion.

\section{CRYPTOGRAPHY}

Cryptography is the intelligent art to make a data as secret. It refers sometimes study or analysis of data in secret type. Cryptography is the best tool for protected communication of image, text, video etc, [1]. Cryptography is exploiting the study of hidden information and makes information as secret. It classifies three different kinds (i) Secret key cryptography (ii) Public key cryptography (iii) Hash functions [4]. In Secret key cryptography, both the dispatcher and beneficiary are used same password or key through their transmission [10]. Secret key cryptography is sometimes known as Symmetric key system. In Public key or asymmetric cryptography, the sender and receiver use a pair of keys for unlock the content. Keys work in pairs matched public and private keys [11]. Hash functions not

depend on the secret key. Encryption and decryption are the two methods to guarantee data as secret in cryptography. Encryption is the process to convert original image data in to some other anonymous structure using a key which is not identified by anyone.

Decryption defines the recover of original data from the encrypted thing. Some of image encryption techniques are used traditionally is Data encryption standard, triple DES, blowfish, advanced encryption standard, scan based, and chaos based etc. [6][12]. An encryption system generally needs a key generation algorithm to arbitrarily produce keys. The traditional cryptography diagram is shown in figure. 1

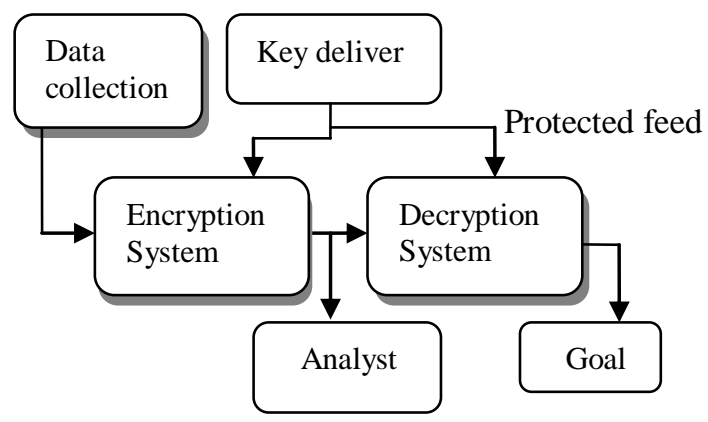

Fig.1. Representation of Cryptosystem 
International Journal of Advanced Research in Computer and Communication Engineering Vol. 3, Issue 10, October 2014

Data passes through the encryption system with a key producer, assigned a right key and same key as send to decryption unit in secure mode. Finally the goal achieves the unique data after decryption.

\section{PROPOSED SCHEME}

Encryption and decryption attain by single key is the previous finest technique of image security. Single key assigned for image encryption and it is encoded.

Then the key is send via secure way for decryption purpose. Subsequently the key is safely received and apply decryption process and obtain original image. The proposed method block diagram is shown in figure 2 .

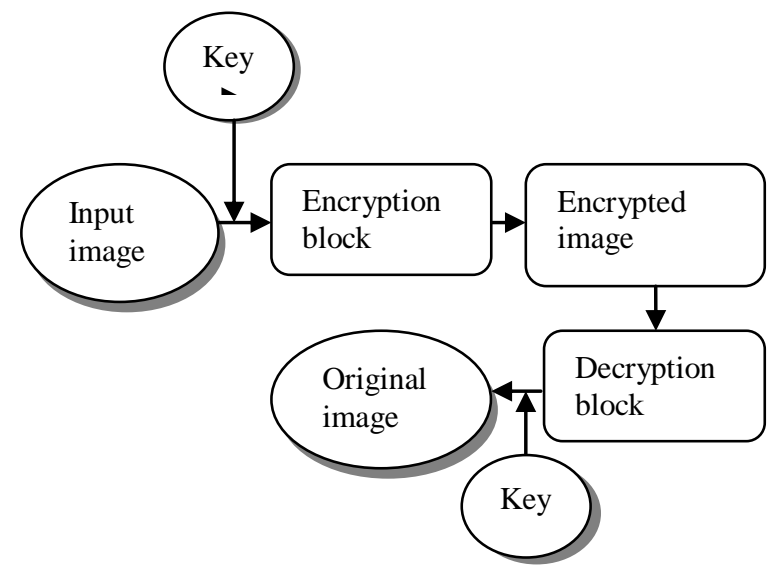

Fig .2. Proposed block diagram

The encrypt part is used to conceal the information of image. This instant no individual can perceive the information. Decrypt part is utilized the secret information to unlock and lay up as original image.

\section{IMPLEMENTATION ALGORITHM}

Two processing are,

(i) Encryption algorithm, (ii) Decryption algorithm

\subsection{Encryption procedure}

1) Input the image

2) Assign a valid key of 256

3) Read the volume of image as matrix

4) Generate matrix of arbitrary numbers

5) Round the random values
6) Apply XOR operation of rounded values

7) Shows encrypted image effect

\subsection{Decryption procedure}

1) Input encrypted image

2) Allot the same key of 256 mixtures

3) Read the size of encrypted image

4) Produce random numbers

5) Encircling the random values

6) Apply Exclusive-OR action of encircle values

7) Show original image

\section{EXPERIMENTAL RESULTS}

The process of the encryption and decryption of images using single key algorithm was verify in Matlab7.0. This algorithm gives better results. The figure shows the input image, encrypted image and decrypted image. The proposed algorithm is applied and tested on a variety of dissimilar formats images.

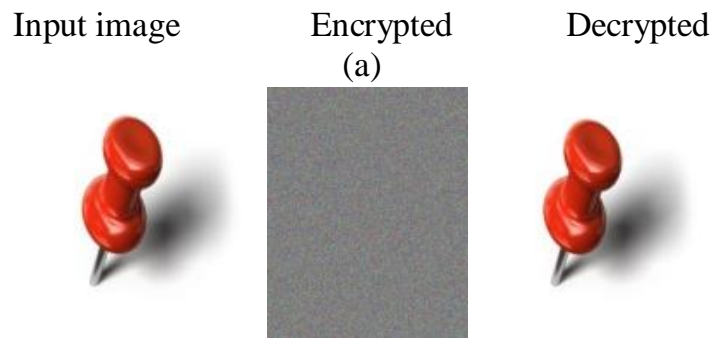

(b)
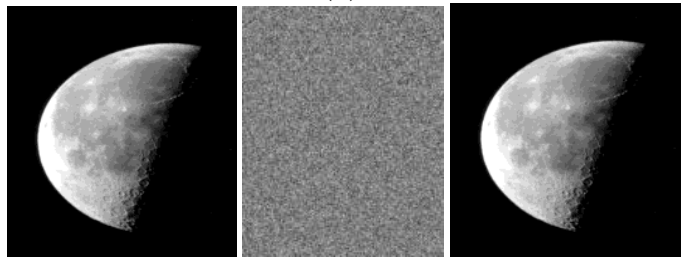

(c)
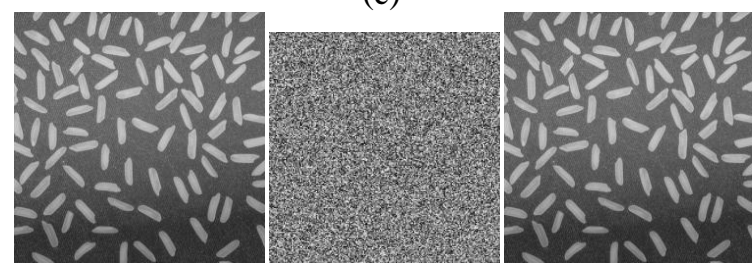

Fig.3.Input, Encrypted and decrypted image

TABLE 1

Results of Selected images

\begin{tabular}{|c|c|c|c|}
\hline $\begin{array}{c}\text { Image } \\
\text { type }\end{array}$ & Dimension & $\begin{array}{c}\text { Actual } \\
\text { Size }\end{array}$ & $\begin{array}{c}\text { Encryption } \\
\text { Size }\end{array}$ \\
\hline (a).jpg & $1050 * 750$ & $158 \mathrm{~KB}$ & $2.25 \mathrm{MB}$ \\
\hline (b) .tiff & $358 * 537$ & $179 \mathrm{~KB}$ & $189 \mathrm{~KB}$ \\
\hline (c).png & $256 * 256$ & $43.5 \mathrm{~KB}$ & $65 \mathrm{~KB}$ \\
\hline
\end{tabular}


International Journal of Advanced Research in Computer and Communication Engineering Vol. 3, Issue 10, October 2014

\section{CONCLUSION}

The image encryption and decryption algorithm is deliberated and realized to afford privacy and protection in communication of the image based data as well as in storage .This proposed algorithm gives a fine results with some draw backs. Whenever image size is increased, it takes more time to encrypt. So further analysis of various techniques of encryption and decryption of image are very much needs to achieve satisfaction.

\section{ACKNOWLEDGMENT}

The author thanks the authorities of Annamalai University for providing the required facilities in order to complete this portion of work.

\section{REFERENCES}

[1] Mayanak Mishra, Prashant Singh, Chinmay Garg "A New Algorithm of Encryption and Decryption of images using chaotic mapping" International Journal of Information and computation technology Volume No.4, Issue No.7, 2014.

[2] Vikas Agarwal ,shruthi agarwal,rajedh deshmukh "Analysis and review of encryption and decryption for secure communication" International Journal of scientific engineering and research, February , 2014

[3] Shetty deepesh sananda,anush karkala "Image encryption and decryption using image gradient technique" International Journal of Emerging technology and advanced engineering", 2014

[4] Rajindar kaur ,Kanwalpreet singh "Comaparative analysis and implementation of image encryption and decryption" International Journal of Computer science and mobile computing, Volume-2, Issue-4 , April, 2013

[5] Shika kuchhcal and ishank kuchhal “ Data security using RSA algorithm in matlab" International journal of innovative research and development, July, 2013.

[6] Pia Sing ,prof.karamjeet singh "Image encryption and decryption using blowfish algorithm in matlab" Interanational journal of scientific and engineering research, July-2013

17] Anju,Babita,Reena and Ayushi Aggarwal " An Approach to improve the data security using encryption and decryption techniques", international journal of information and computation technology,2013

[8] Quist-Aphetsi kester "Image encryption based on the TGB PICEL transposition and shuffling" ,International journal of computer network and information security",2013

[9] Rinki Pakshwar,vijay kuamr,trivedi,vineet richhariya " A survey on different image encryption and decryption techniques", IJCSIT, 2013

[10] Dr.j.Abdul jaleel,jisha mary Thomas " Gurading images using a symmetric key cryptographic techniques : Blowfish algorithm" ,international journal of engineering and innovative technology,august, 2013

[11] Harpeet sing,Dr.naveen dhillon,sukhpreet singh bains "A new approach for image cryptography techniques" IJCOT,Oct,2013

[12] Rinkee gupta " color image encryption and decryption using scan approach"IJSHRE,2013

[13] Bhaskara reddy et.al "An effective algorithm of encryption and decryption of images using random number generation technique and huffman coding, IJCTA,Nov,2013

[14] Hiral rathod,mahendra sing, sanjay kumar Sharma " Design and implementation of image encryption algorithm by using block based symmetric transformation algorithm, IJCTEE

[15] Husamettin Uysal,sinem kurt tulay " Automatic decryption of images through artificial neural networks" ,Trends in innovative computing ,2012

[16] Gamil R.S.Qaid,sanjay n.Talbar, “ Encryption and decryption of digital image using color signal”, ,IJCSI,2012

[17] Manoj.B.ManjulaN.Harihar "Image Encryption and decryption using AES", International journal of engineering and advanced technology ,June,2012

[18] Amrita sahu, et.al, "Proposed method of cryptographic key generation for securing digital images , International journal of advanced research in computer science and software engineering 